\title{
Infectious bronchitis virus variants in chickens: evolution, surveillance, control and prevention
}

\author{
Rodrigo A. Gallardo ${ }^{\mathrm{a}^{*}}$
}

\begin{abstract}
Infectious bronchitis is a disease of the upper respiratory tract of chickens caused by a Gammacoronavirus (infectious bronchitis virus, IBV). Severe economic losses are caused by IBV due to a reduction in egg production and/or egg quality in layers in addition to poor feed conversion and increased condemnations in broiler chickens. The extreme variability of this virus is in part due to its RNA genome, which predisposes it to mutations and generates genetic variation. In addition, recombination events add to the variability of this virus. IBV variability was first described in 1956 by Jungherr. Since then, dozens of serotypes and hundreds of genotypes have been reported. Variant IBV strains are those that, can escape from the immunity generated by conventional strains, despite not being fully different from conventional strains affecting a geographic region. At the genomic level, these differences can be equal or greater than 5\% of the hypervariable region of the $\mathrm{S} 1$ gene. These variant strains are usually restricted to geographic regions and most of the time are transient, reason why diagnostics and epidemiological surveillance are crucial to determine their existence and persistence. The main goal of surveillance is to assist the development of efficient preventative measures in the field. This review aims to critically analyse the literature related to IBV variability and judiciously comment and discuss on how to better prevent this poultry endemic disease.

Key words: IBV, variants, variability, evolution, surveillance.
\end{abstract}

\section{INTRODUCTION}

Understanding factors that might play a role in the generation of infectious bronchitis variants is crucial in order to perform surveillance, prevent their occurrence and establish prevention strategies. These factors involve inherent viral characteristics, management, vaccines and vaccination, immunity and concomitant infectious diseases. The purpose of this review is to merge research findings with clinical observations and laboratory expertise on the generation, diagnostic, surveillance and prevention of infectious bronchitis variant strains in commercial chicken production.

The infectious bronchitis coronavirus (IBV) is the causative agent of one of the most economically important diseases in modern poultry production, infectious bronchitis (IB), in chickens. The disease was first described by Schalk and Hawn in 1931. Nowadays the disease is endemic in most of the countries that possess a developed commercial poultry industry. Its global economic impact has been estimated as the second most damaging poultry disease after highly pathogenic avian influenza (TAFS-Forum 2011). The clinical picture is associated with upper respiratory tract infection characterised by conjunctivitis, tracheitis and loss of ciliary movement in the trachea. In addition, reproductive effects such as drop in egg production and alterations in the egg internal and

Received: 29.10.2020.

Accepted: 02.03.2020.

a Department of Population Health and Reproduction, School of Veterinary Medicine, University of California, California, USA.

*Corresponding author: R Gallardo; 1089 Veterinary Medicine Dr. VM3B, Davis, CA 95616, USA; ragallardo@ucdavis.edu external quality can be seen in layer and breeder birds. If layer chicks get exposed early in their lives the virus can infect the oviduct, altering its normal development and inducing false layers (Broadfoot et al 1956, Crinion and Hofstad 1972, Gallardo et al 2019). Some IBV strains have kidney tropism inducing nephritis and urate deposition (Winterfield and Albassam 1984, Albassam et al 1986) while others have been associated to enteric disorders (El-Houadfi et al 1986, Hauck et al 2016). Secondary infections are common in meat-type birds affected by IBV inducing airsacculitis and increasing condemnations at the processing plant.

\section{INFECTIOUS BRONCHITIS VIRUS}

IBV is a single-stranded, positive sense RNA virus of the Coronaviridae family, genus Gammacoronavirus ${ }^{1}$. The viral genome comprises two untranslated regions (UTR's) in its 5' and 3' ends, two overlapping reading frames (ORF's) encoding the structural polyproteins 1a and $1 \mathrm{ab}$, and the region encoding the main structural proteins i.e. spike (S), envelope (E), membrane (M) and nucleocapsid (N). Finally, two accessory genes ORF 3 and ORF 5 encode for proteins $3 a, 3 b$ and $5 a$ and $5 b$ (Ziebuhr et al 2000). The $\mathrm{S}$ protein is located on the surface of the virus external membrane. During binding to the host cell, the $S$ protein is cleaved on a cleavage site rich in basic bases into the amino-terminal S1 ( 535 amino acids) and the carboxy-terminal S2 ( 627 amino acids) (Cavanagh et al 1986). The $\mathrm{S} 1$ portion of the $\mathrm{S}$ protein of IBV is responsible for viral attachment to host cells, virus variability and eliciting neutralizing antibodies in

1 http://www.talk.ictvonline.org/taxonomy/ 
chickens (Cavanagh 1983, Cavanagh and Davis 1986, Kusters et al 1987, Casais et al 2003). The S1 displays the most genetic and phenotypic variability among different IBV strains (Cavanagh 1983, Cavanagh and Davis 1986, Kusters et al 1987) and is therefore the best target to assess variability. Variant IBV strains are those that, despite not being fully genetically different from conventional strains affecting a geographic region, are phenotypically different and escape from the immunity generated by conventional serotype-specific vaccines. At the genomic level, these differences can be greater or equal to $5 \%$ in the hypervariable region of the $\mathrm{S} 1$ gene. These variant strains are usually restricted to geographic regions and most of the time are transient (Gallardo et al 2016), reason why diagnostics and epidemiological surveillance are crucial to determine their existence and persistence. This strategy helps planning preventative measures in the field.

\section{IBV EVOLUTION}

Viral evolution depends on two separated and independent mechanisms described by Mayr (Mayr 1988): (a) Generation of diversity, in which genetic/phenotypic variants are generated and serve as material for (b) selection, the virions generated after the replication process are released in the environment, and the survivors will serve as the genetic pool for subsequent generations (Gallardo et al 2010). IBV variability is generated by insertions, deletions and point mutations in addition to recombination events (Toro et al 2012). Jungherr and collaborators reported viral variability between IBV isolates for the first time in 1956. The described serological variations differentiated Massachusetts and Connecticut IBV serotypes (Jungherr et al 1956). IBV selection has been proved previously by different research groups (McKinley et al 2008, van Santen and Toro 2008). The rapid evolution ability is what makes IBV highly successful in the environment and is the reason why IBV continues to spread and circumvent vaccination programs used in the poultry industry (Toro et al 2012). Nowadays, we recognise the existence of dozens of IBV serotypes and even more genotypes and variants. Variants have been detected all over the world: Latin America, associated with kidney lesions (Hidalgo et al 1986); Africa, associated with swollen head syndrome (Morley and Thomson 1984); Egypt, associated with enteric disease (El-Houadfi et al 1986). In Asia, variants have been present since at least 1979 (Lohr 1988). In Australia, where evolution has been independent from other countries in the world (Ignjatovic et al 2006), variant IBV strains have been isolated since 1960 (Cumming 1963).

The introduction of exotic genotypes into a geographic region or country can increase the local genetic pool of IBV strains. This situation increases the chances of genetic recombination with local IBV strains and potentially generates IBV variants. The introduction of these IBV strains can be accidental, via contaminated poultry products and lack of biosecurity, and/or premeditated. The most common premeditated introduction is caused when vaccines from exotic genotypes are introduced as a tool for controlling IBV outbreaks caused by variant strains. There are several examples of generation of variant IBV strains after the premeditated introduction of exotic genotypes. This review will focus on two cases. The first example is the event reported by Lee and Jackwood, where they describe the generation of the IBV genotype GA98 after the introduction and use of the DE072 vaccine. The IBV GA98 was the causal agent of extensive and costly outbreaks of IB in broilers in the state of Georgia (Lee and Jackwood 2001). The second example is the introduction of the IBV vaccine type 793B, 4/91 or 1/96, a UK-originated variant, to countries in Europe (Franzo et al 2014, Moreno et al 2017), Middle East (Tatar-Kis et al 2014) and Latin America (Sesti et al 2014, de Wit et al 2017). The reported consequences in Italy and Spain have been the recombination between local strains and the introduced vaccine (Moreno et al 2017).

\section{VACCINES AND THEIR USE}

Vaccines capable of inducing cross protection against different genotypes of IBV are of paramount economic and practical importance (Gelb 2018). Some commercially available vaccines are heterogenous, meaning that the predominant subpopulations in the vaccine are diverse and do not induce protective immune responses in chickens (Ghetas et al 2014). The best example of these heterogeneous vaccines is the ArkDPI vaccine. ArkDPI was originally isolated from the Delmarva peninsula and partially attenuated at the University of Delaware by 50 passages in embryonated eggs (Gelb and Cloud 1983). Then, the strain was distributed to different vaccine companies where they were further attenuated to produce different vaccines. The heterogeneity of the $S$ gene in vaccines like ArkDPI might play the role of a virulent strain in terms of genetic variability, resulting in the emergence of new variants after replication, generation of diversity and selection. In addition, recombination of vaccine strains with local strains might also play a role. ArkDPI vaccines showing higher heterogeneity have been associated with respiratory signs and tracheal damage (Ndegwa et al 2012). Studies in variant IBV variability have shown different levels of heterogeneity in different IBV genotypes (Gallardo et al 2016). It is important to understand the heterogeneity of variant IBV strains before selecting them as vaccine candidates. Vaccine homology will also play a role. Heterologous vaccines will either provide no protection or, at best, partial protection. If partial protection is achieved, it will ameliorate clinical signs but will not reduce shedding of challenge/field virus. High loads of wild-type IBV strains combined with vaccine viruses creates the perfect scenario for variant virus generation. In summary, as it has been reported in the literature, live 
attenuated vaccine usage has a major role in the genetic profile of IB strains isolated in the field (Jackwood and Lee 2017).

Other than vaccine selection, vaccine application is crucial to avoid variant IBV generation. Currently available live attenuated IBV vaccines are mostly applied in the hatchery at day of age and by spray or drinking water between 10 to 15 days of age in broilers and at least 3 to 4 times in layers before the laying onset. Massive application strategies for IBV vaccination are partially inefficient and usually result in vaccination failure (Jordan 2017). An example of partly ineffective vaccination methods are spray cabinets. Although the spray vaccination processing does not seem to damage the IBV virions, there is a significant titer reduction, most likely associated with the mechanical force applied to the virus particle during the vaccination process (Roh 2014). In addition, viruses are greatly wasted in the environment, especially when volumes as low as $7 \mathrm{ml}$ per chick box are used (Jordan 2016). These issues are responsible for poor coverage during spray cabinet vaccination. Similar issues happen when using drinking water vaccination in the field, where poor vaccine coverage allows vaccine recirculation and rolling reactions. These rolling reactions allow the virus to mutate in every replication cycle while poor coverage allows the entrance of field viruses into the flock, creating recombination opportunities and subsequently generation of variant strains. All these problems are aggravated if half or quarter doses are applied.

\section{POPULATION IMMUNE STATUS}

Population immune status plays a crucial role in the evolution of IBV, not only because of the effect of vaccination in selective pressure but also because of immunodepression that will allow the virus to evolve freely in the affected population. Numerous epidemiological studies using conventional and molecular virology techniques have demonstrated the capabilities of IBV to circumvent vaccination programs which have been implemented since 1950 (Toro et al 2012). In particular, it is interesting to analyze Ark-serotype attenuated vaccines, where viral populations different from the vaccine emerge after vaccination causing disease and poor immune responses in vaccinated chicken flocks (McKinley et al 2008, Toro et al 2012). In the absence of immune pressure due to vaccination, IBV nucleotide changes in the $\mathrm{S} 1$ gene occur at a rate of $10^{-3}$ substitutions per site per year (Cavanagh et al 1998). GA98, which emerged due to the use of DE072 vaccine, showed mutation rates of $10^{-2}$ substitutions per site per year in the hypervariable region (HVR) of S1(Lee et al 2001). Immunocompetent chickens show normal immune responses that are able to restrict viral replication and limit generation of genetic variants for selection. In chickens showing less than optimal immune responses, the vaccine viruses are able to replicate in a larger number of individuals and viral populations different from the challenge strain may become predominant (Gallardo et al 2012, Toro et al 2012). This phenomenon can particularly happen when chickens are infected by highly prevalent and ubiquitous immunosuppressant viruses, i.e. chicken anemia (CAV) and infectious bursal disease virus (IBDV). Infectious bronchitis infection course in CAV or IBDV immunosuppressed flocks is longer, the clinical signs are more severe and the virus is able to persist for longer periods in the environment, facilitating diversity generation and subsequently emergent variant IBV strains (Gallardo et al 2012).

\section{DETECTION, SURVEILLANCE, CONTROL AND PREVENTION}

\section{DETECTION AND SURVEILLANCE OF IBV}

While we are very informed about the IBV strains affecting chickens in the U.S. and most of the countries in the E.U. due to continuous surveillance, there is lack of information in several countries in the world. In countries where surveillance is performed, most of the genotypes detected are not indigenous variants (de Wit et al 2010), but rather genotypes derived from the evolution of prevalent viruses and vaccines used in the region. Poor diagnostic and surveillance capabilities are a reality in most Latin American and African countries and, where these tools are available, it is very common to see errors in technique and result interpretation (unpublished data). Accurate diagnosis and targeted surveillance is crucial to adequately prevent and control IBV variant rise and its detrimental effects (Gallardo et al 2016). Other than orienting in the vaccine selection and vaccination strategy, accurate diagnosis will drive efforts to impede variant generation. IBV diagnostics are based on: (1) detection of the viral genome by molecular methods, such as reverse transcriptase polymerase chain reaction (RT-PCR) or RT quantitative PCR (RT-qPCR), targeting conserved genes such as N or M (Gallardo et al 2010, de Quadros 2012) and (2) conventional virology methods, such as virus isolation, serology and virus neutralization (Villegas and Alvarado 2008). A combination of these techniques is used in several laboratories around the world for diagnosing the disease. For surveillance, serum neutralisation in embryonated eggs, haemagglutination inhibition and sequencing of a portion or full length $S$ gene amplified by RT-PCR are the most commonly used techniques (Villegas and Alvarado 2008, Gallardo et al 2010). Serum neutralization is used to type IBV using serotype-specific antibodies. However, hyperimmune sera are not readily available when new IBV variants arise. In addition, serum neutralisation test is expensive due to the use of a large number of SPF eggs. A similar problem is observed with HI, where specific reagents are needed. In addition, $\mathrm{HI}$ requires the treatment of the IBV isolate with neuraminidase since IBV does not have haemagglutination 
capabilities naturally. Nowadays, the most commonly used technique is the RT-PCR to amplify the HVR of the $\mathrm{S} 1$ gene followed by sequencing of the amplicon, with or without prior virus isolation (Gallardo et al 2010). Virus isolation, if successful, increases the number of viral particles and consequently the amount of template for the polymerase reaction. The drawback of previous virus isolation is that in vitro replication allows viral mutations in the IBV genome, increasing variability during the virus adaptation to the embryonated egg. RT-PCR directly from chicken specimens followed by sequencing, even though less sensitive, is the best method to perform surveillance. This methodology will provide genetic information about the wild-type virus without the variability added during virus isolation. The nucleotide heterogeneity of S1 is largely contained within three different HVR's. Those HVR's are located in amino acids 38 to 67,91 to 141 and 274 to 387 (Cavanagh et al 1988). Complete or partial analysis of the $\mathrm{S} 1$ has been used to determine IBV genotypes. Currently, nearly 100 genotypes have been recognised for causing impact on the commercial poultry industry. Some are restricted to specific geographic areas (Gallardo et al 2016).

The size of the S1 fragment amplified by RT-PCR for surveillance is of major importance. While using the full $\mathrm{S} 1$ is the best choice because it allows the analysis of all three HVR's, this technique is less sensitive than amplifying a shorter fragment of $\mathrm{S} 1$ and requires a considerable amount of well-preserved viral RNA in the sample. On the other hand, while amplification of small segments (between 300 and 500 bp) increases the test sensitivity, short sequences overlook variations in other portions of the HVR's or recombination sites (Moreno et al 2017). While not as accurate as using the full $\mathrm{S} 1$ gene, the amplification of a segment of 750 nucleotides has been proven to be adequate for IBV surveillance and evolution studies et al 2012). Inconsistencies in phylogenetic analyses makes IBV genotyping and characterisation difficult. Valastro proposed a classification system using full $\mathrm{S} 1$ sequences (Valastro et al 2016). The reasoning behind this classification is that there is lack of consistency when using only one or two of the three HVR's of the S1gene in the analysis. In addition, the spatial component of the characterisation shows evidence that most of the current strains originated from old lineage IBV's and their rapid evolution assures the discovery of new genetic variants in the future. Full genome characterisations are useful when available. The information provided by whole genome sequencing should confirm the partial or complete analysis of the $\mathrm{S}$ gene and provide further information on other genes. Variability of the outer viral proteins such as the spike and envelope are of particular interest.

\section{SURVEILLANCE INTERPRETATION}

The routinely performed genotypic surveillance of IBV involves molecular amplification and sequencing of a portion of $\mathrm{S} 1$ or the complete $\mathrm{S} 1$ gene. These sequences are curated and compared with sequences located in databases such as GenBank. The sequences should be compared in phylogenetic trees with the most commonly used vaccines and strains isolated in the geographic area of interest. The size of the segments compared are of major importance. Short sequences do not account for all HVR's and might provide inaccurately high homologies when compared to other sequences in the database, leading to wrong interpretations. In addition, comparing segments of different lengths can generate high identities with low query coverages, resulting in biased homologies that need to be considered in the interpretation. If the coverage is low, the confidence of the analysis might be at risk. The detection of a genetic variant does not imply that this virus is the causal agent of problems in the field. Surveillance data should be correlated with clinical signs, pathology, management, field data and vaccination records. Not all variants acting in a geographic region require the preparation of autogenous vaccines since a percentage of them are transient and restricted to a limited geographic area. It is important, other than performing molecular epidemiology using phylogenetic trees, to associate these trees with pathological observations in the field, perform virus isolation and biological characterisation in chickens. If the viruses are highly pathogenic or cause severe productive losses, vaccine protection studies are recommended. The objective is to test protection elicited by commercially available vaccines. If the protection provided by the available vaccines is not adequate, studies to assess variability of the detected variant should be performed to determine if formulating a homologous vaccine to prevent outbreaks is safe (Gallardo et al 2016). Sometimes IBV variants that are not clearly associated with disease are detected. In this case, it is recommended to continue with close epidemiological surveillance. Generation of databases for specific regions is desired, since they allow for better interpretation of results (Gallardo et al 2016).

\section{CONTROL OF IMMUNOSUPPRESSIVE VIRAL DISEASES}

Immunosuppression in poultry can be caused by several factors, including stress, nutritional deficiencies, mycotoxins and viral diseases (Hoerr 2010). The main viral immunosuppressive diseases reportedly linked with IBV cases in the field are IBDV and CAV. IBV cases in broiler chickens usually occur between 35 and 49 days of age (figure 1) and are potentially associated with immune deficiencies (Toro et al 2006, Gallardo et al 2016). As a confirmatory experiment, bursa of Fabricius samples were collected from a representative number of flocks in a broiler integrator in the U.S. Each bursa was divided in half, one half was used for IBDV viral load measurement by RT-qPCR and the other half was used for bursal histomorphometry to evaluate lymphoid depletion (Gallardo 2018) (figure 2). 
Results showed a peak of IBD viral load between 25 to 30 days of age, followed by a peak of lymphoid depletion in the bursa around day 35 of age. These results correlate with the reported age of highest incidence of IBV cases in broiler chickens (figure 1) (Gallardo et al 2016). This correlation was previously reported by Toro et al (2006) where they measured lymphoid depletion scores of bursa and thymus sections in 322 cases of diagnosed IB between 1997 and 2002 in Alabama. In that project, peaks of lymphoid depletion associated with IBDV and CAV in bursa and thymus, respectively, were found in broilers between 30 and 40 days of age (Toro et al 2006). IBDV and CAV immunosuppression can be prevented by vaccination strategies. An efficient vaccination strategy for IBDV prevention must involve hyperimmunisation of breeders using live attenuated or recombinant and inactivated vaccines in order to protect chickens until approximately 3 to 4 weeks of age. In addition, vaccination of the progeny with recombinant, live attenuated or antigen-antibody complex IBDV vaccines provide appropriate protection until approximately 5 to 6 weeks of age in broilers (Gallardo 2018). For CAV, an effective preventative strategy is to generate adequate immune response in the breeders to impede vertical transmission and to transfer maternal antibodies to the chicks. For that, an adequate vaccine should be selected and the induced immunity should be

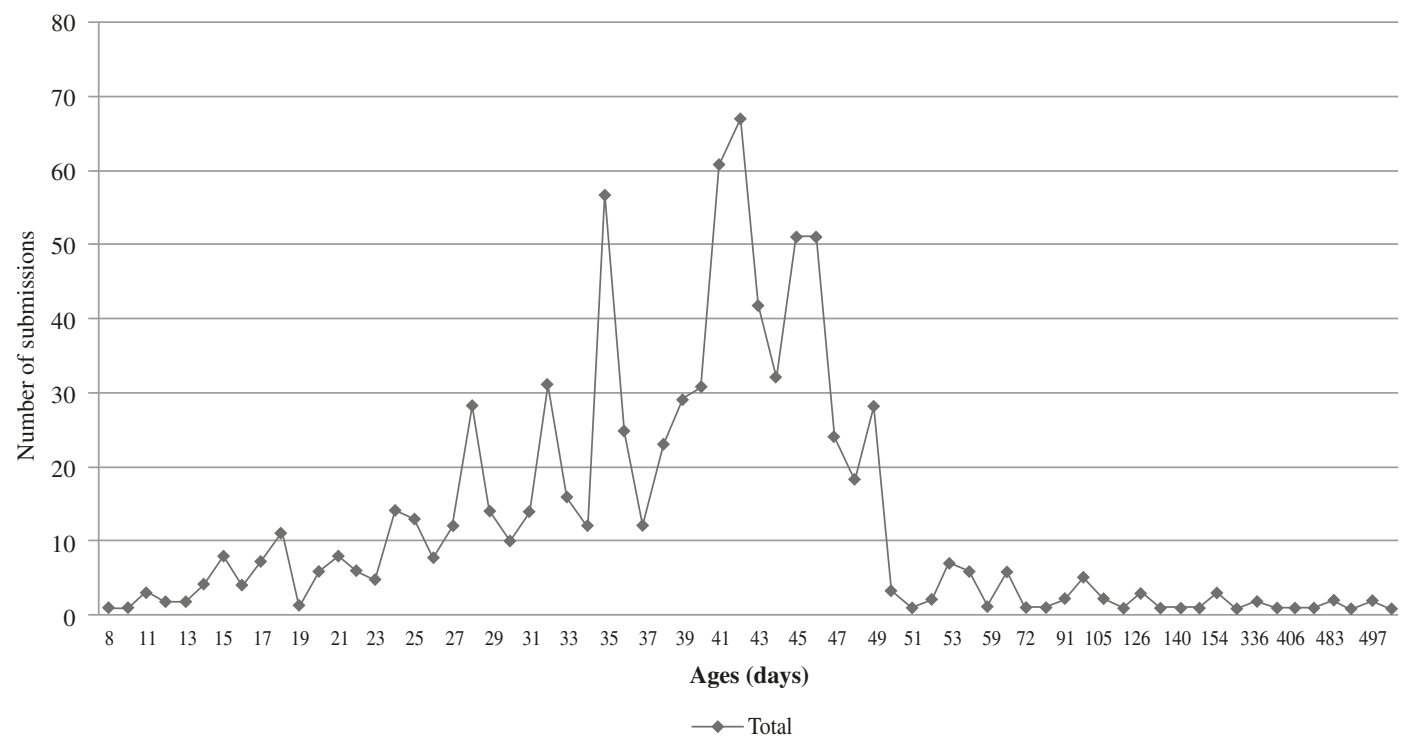

Figure 1. Age of occurrence of IBV respiratory cases in broilers and layers (n:1444) in California between 1997 and 2012.

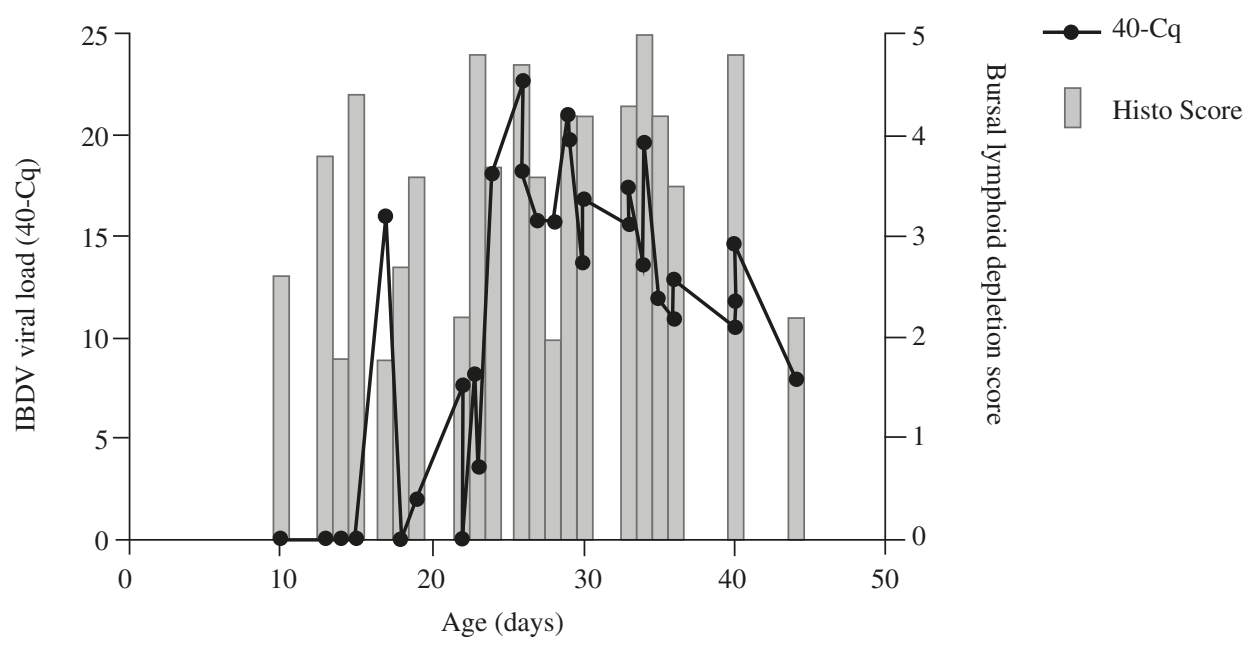

Figure 2. Bursa of Fabricius lymphocyte depletion scores from 0 to 5. (0: no lymphoid depletion to 5: highest level of lymphoid depletion) and viral load in bursas expressed as 40-Cq. These data were obtained from commercial broiler chickens at different days of age starting at 10 days and ending at 43 days. 
periodically assessed (Gallardo 2018). There are commercial ELISA kits available for CAV antibody assessments. Serum samples diluted 1:100 allow clear dispersion of the data points (Toro 2015). The results should be analysed considering $\mathrm{S} / \mathrm{N}$ ratio limit values of 0.2 and 0.8 . Values below 0.2 represent protective titers, values between 0.2 and 0.8 represent low titers and values above 0.8 represent non-protected chickens or negative titers (Toro 2015). Even though it has not been reported as a direct cause of increased IBV cases, Marek's disease virus (MDV) can cause immunosuppression, affecting both humoral and cellular immune responses (Gimeno and Schat 2018). The MDV immunosuppression pathogenesis is very complex, poorly understood and in many cases under diagnosed. MDV vaccination protects against some aspects of viral immunosuppression but certainly not all (Gimeno and Schat 2018).

\section{IBV MANAGEMENT}

Proper management is a key component of a disease-free flock. Chickens can be exposed to stressors and infectious diseases that impair innate and acquired immunity, eroding general health and welfare (Hoerr 2010). Any stress caused by poor management can affect the immune system and the consequences can be linked to upper respiratory tract infections (Hoerr 2010). An example of poor management is poor ventilation. If intensive productive units are poorly ventilated, the incidence of respiratory diseases increases. Increased particulate matter and ammonia in the air (Shepherd et al 2015, Zhao et al 2015), in addition to the increased concentration of Gram-negative bacteria in the environment (Zhao et al 2016), can cause irritation, inflammation and changes in the microbiota, inducing complex upper respiratory diseases (figure 3) (Gallardo 2018). Addressing the presence of risk factors and understanding their interaction is essential for a successful management and consequently optimal health and welfare. In addition, genetics and nutrition have a major role in an efficient production system (Hoerr 2010). Food-borne mycotoxins and suboptimal nutrition can diminish immune responses, particularly the innate immunity to pathogens (Hoerr 2010). Biosecurity is the first barrier for pathogen introduction in poultry flocks. Lack of or flawed biosecurity facilitate the introduction of IBV, immunosuppressive pathogens (CAV, IBDV, MDV, reoviruses and adenoviruses) and other respiratory viruses such as avian influenza, Newcastle disease and avian metapneumoviruses. These upper respiratory tract viruses affect the ciliated epithelium and mucous glands of sinuses and tracheas, predisposing the entrance of more pathogens.

In conclusion, IBV is highly variable and variants arise because of the constant evolution of this virus. There are factors that predispose the virus to more variability, such as the introduction of exotic genotypes, poor vaccine selection and application and immunosuppressive diseases.

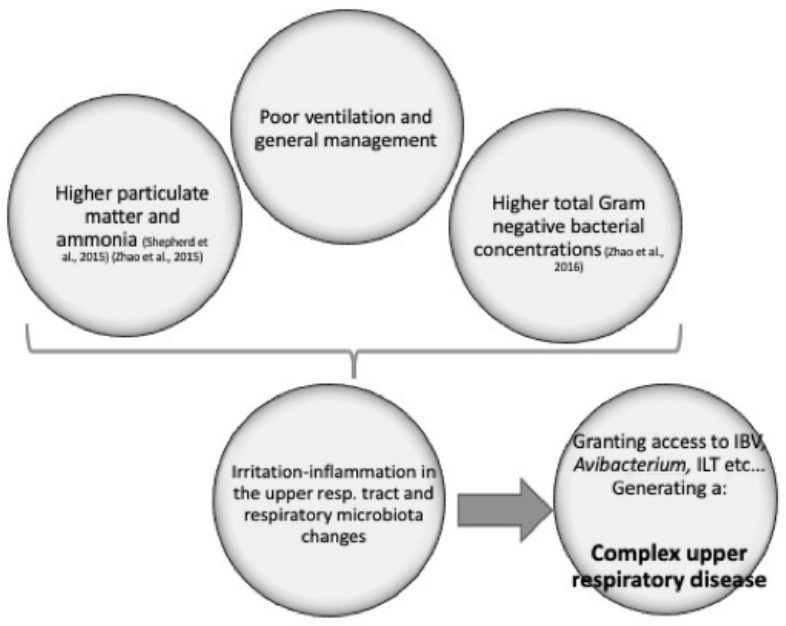

Figure 3. Effects of poor management in the air quality and upper respiratory tract of chickens, pathogenesis of complex upper respiratory disease.

Diagnosis, detection and surveillance are necessary to investigate and properly analyse the circulating IBV genotypes. This understanding depends on appropriate interpretation of results and assists the development of suitable preventative strategies and vaccination decisions. In addition, good practices, management and adequate control of immunosuppressive diseases help preventing the arise of variant IBV strains. Prevention of endemic diseases in poultry is based on properly using the available diagnostic and surveillance tools and interpreting the results obtained. When these tools are not properly used, misleading information is shared, and deceptive epidemiological data is reported. These events can affect future preventative strategies and predictions on IBV evolution.

\section{ACKNOWLEDGEMENTS}

I would like to acknowledge Dr. A.P. Da Silva for critical review of this manuscript.

\section{REFERENCES}

Albassam MA, Winterfield RW, Thacker HL. 1986. Comparison of the nephropathogenicity of four strains of infectious bronchitis virus. Avian Dis 30, 468-476.

Broadfoot DI, Pomeroy BS, Smith WM. 1956. Effects of infectious bronchitis in baby chicks. Poultry Sci 35, 757-762.

Casais R, Dove B, Cavanagh D, Britton P. 2003. Recombinant avian infectious bronchitis virus expressing a heterologous spike gene demonstrates that the spike protein is a determinant of cell tropism. J Virol 77, 9084-9089.

Cavanagh D. 1983. Coronavirus IBV: structural characterization of the spike protein. J Gen Virol 64, 2577-2583.

Cavanagh D, Davis PJ. 1986. Coronavirus IBV: removal of spike glycopolypeptide $\mathrm{S} 1$ by urea abolishes infectivity and haemagglutination but not attachment to cells. J Gen Virol 67, 1443.

Cavanagh D, Davis PJ, Pappin DJC, Binns MM, Boursnell MEG, et al. 1986. Coronavirus IBV: Partial amino terminal sequencing of spike 
polypeptide S2 identifies the sequence Arg-Arg-Phe-Arg-Arg at the cleavage site of the spike precursor propolypeptide of IBV strains Beaudette and M41. Virus Res 4, 133-143.

Cavanagh D, Davis PJ, Mockett APA. 1988. Amino acids within hypervariable region 1 of avian coronavirus IBV (Massachusetts serotype) spike glycoprotein are associated with neutralization epitopes. Virus Res 11, 141-150.

Cavanagh D, Mawditt K, Adzhar A, Gough RE, Picault JP, et al 1998. Does IBV change slowly despite the capacity of the spike protein to vary greatly? Enjuanes L, Siddell SG, Spaan W (eds). Coronaviruses and Arteriviruses. Springer, Boston, MA, US, Pp 729-734.

Crinion RAP, Hofstad MS. 1972. Pathogenicity of four serotypes of avian infectious bronchitis virus for the oviduct of young chickens of various ages. Avian Dis 16, 351-363.

Cumming RB. 1963. Infectious avian nephrosis (uraemia) in Australia. Australian Vet J 39, 145-147.

de Quadros VL. 2012. Das infektiöse bronchitis virus (IBV): molekularbiologische untersuchungen zur diagnostik und zum vorkommen sowie zur pathogenität des genotyps IBV QX in spezifisch pathogenfreien (SPF) broilern. Doctorate dissertation, Freien Universität Berlin, Berlin, Germany.

de Wit J, Cook J, van der Heijden H. 2010. Infectious bronchitis virus in Asia, Africa, Australia and Latin America: history, current situation and control measures. Brazilian J Poult Sci 12, 97-106.

de Wit JJ, Dijkman R, Guerrero P, Calvo J, Gonzalez G, et al. 2017. Variability in biological behaviour, pathogenicity, protectotype and induction of virus neutralizing antibodies by different vaccination programmes to infectious bronchitis virus genotype Q1 strains from Chile. Avian Pathol 46, 666-675.

El-Houadfi M, Jones RC, Cook J KA, Ambali AG. 1986. The isolation and characterisation of six avian infectious bronchitis viruses isolated in morocco. Avian Pathol 15, 93-105.

Franzo G, Naylor CJ, Lupini C, Drigo M, Catelli E, et al. 2014. Continued use of IBV 793B vaccine needs reassessment after its withdrawal led to the genotype's disappearance. Vaccine 32, 6765-6767.

Gallardo RA, van Santen VL, Toro H. 2010. Host intraspatial selection of infectious bronchitis virus populations. Avian Dis 54, 807-813.

Gallardo RA, van Santen VL, Toro H. 2012. Effects of chicken anaemia virus and infectious bursal disease virus-induced immunodeficiency on infectious bronchitis virus replication and genotypic drift. Avian Dis 41, 451-458.

Gallardo RA, Aleuy O, Pitesky M, Sentíes-Cué CG, Abdelnabi A, et al. 2016. Variability assessment of California infectious bronchitis virus variants. Avian Dis 60, 424-429.

Gallardo RA. 2018. Cepas variantes de bronquitis infecciosa: Origen, prevención y control. Proceedings Seminario AMEVEA, Athens, GA, USA.

Gallardo RA, Da Silva AP, Mendoza-Reilley A, Alvarado I, Giroux C, et al. 2019. False layer syndrome caused by IBV, genetic characterization and pathobiology insights. American association of avian pathologists annual meeting, Washington DC, USA.

Gelb J, Cloud SS. 1983. Effect of serial embryo passage of an Arkansastype avian infectious bronchitis virus isolate on clinical response, virus recovery, and immunity. Avian Dis 27, 679-687.

Gelb Jr J. 2018. Protection provided by multivalent infectious bronchitis virus vaccines against challenge with current U.S. strains and variants. U.S. Poultry and Egg Association Project Report.

Ghetas A, Thaxton G, Breedlove C, van Santen V, Toro H. 2014. Effects of adaptation of infectious bronchitis virus Arkansas attenuated vaccine to embryonic kidney cells. Avian Dis 59, 106-113.

Gimeno IM, Schat KA. 2018. Virus induced immunosupression in chickens. Avian Dis. 62, 272-285

Hauck R, Gallardo RA, Woolcock PR, Shivaprasad H. 2016. A coronavirus associated with runting stunting syndrome in broiler chickens. Avian Dis 60, 528-534.

Hidalgo H, Gallardo R, Toro H. 1986. Antigenic and pathogenic properties of three isolates of infectious bronchitis virus obtained from vaccinated chickens. $J$ Vet Med 33, 23-35.
Hoerr FJ. 2010. Clinical aspects of immunosuppression in poultry. Avian Dis 54, 2-15.

Ignjatovic J, Gould G, Sapats S. 2006. Isolation of a variant infectious bronchitis virus in Australia that further illustrates diversity among emerging strains. Arch Virol 151, 1567-1585.

Jackwood MW. 2012. Review of infectious bronchitis virus around the world. Avian Dis 56, 634-641.

Jackwood MW, Lee DH. 2017. Different evolutionary trajectories of vaccine-controlled and non-controlled avian infectious bronchitis viruses in commercial poultry. PloS one 12, e0176709.

Jordan BJ. 2016. Spray application of infectious bronchitis virus vaccines in the hatchery: How efficient are we?. Proceedings XXIV Congreso de Avicultura Centroamericano y del Caribe, Antigua, Guatemala.

Jordan BJ. 2017. Vaccination against infectious bronchitis virus: A continuous challenge. Vet Micro 206, 137-143.

Jungherr EL, Chomiak TW, Luginbuhl RE. 1956. Immunologic differences in strains of infectious bronchitis virus. $60^{\text {th }}$ annual meeting of the US Livestock Sanitary Association, Chicago, USA.

Kusters JG, Niesters HGM, Bleumink-Pluym N, Davelaar FG, Horzinek MC, et al. 1987. Molecular epidemiology of infectious bronchitis virus in the Netherlands. J Gen Virol 68, 343.

Lee, C-W, Hilt DA, Jackwood MW. 2001. Identification and analysis of the Georgia 98 serotype, a new serotype of infectious bronchitis virus. Avian Dis 45, 164-172.

Lee C-W, Jackwood MW. 2001. Origin and evolution of Georgia 98 (GA98), a new serotype of avian infectious bronchitis virus. Virus Res, 80, 33-39.

Lohr LE. 1988. Infectious bronchitis in New Zealand, Asia, East Europe. Proceedings of the $1^{\text {st }}$ International Symposium on Infectious Bronchitis, Rauischholzhausen, Germany, Pp. 70-75.

Mayr E. 1988. Toward a new philosophy of biology: Observations of an evolutionist. Harvard University Press, Massachusetts, USA.

McKinley ET, Hilt DA, Jackwood MW. 2008. Avian coronavirus infectious bronchitis attenuated live vaccines undergo selection of subpopulations and mutations following vaccination. Vaccine 26 , $1274-1284$

Moreno A, Franzo G, Massi P, Tosi G, Blanco A, et al. 2017. A novel variant of the infectious bronchitis virus resulting from recombination events in Italy and Spain. Avian Pathol 46, 28-35.

Morley AJ, Thomson D K. 1984. Swollen-head syndrome in broiler chickens. Avian Dis 28, 238-243.

Ndegwa EN, Joiner KS, Toro H, van Ginkel FW, Santen VL. 2012. The proportion of specific viral subpopulations in attenuated Arkansas delmarva poultry industry infectious bronchitis vaccines influences vaccination outcome. Avian Dis 56, 642-653.

Roh H-J, Jordan BJ, Hilt DA, Ard MB, Jackwood MW. 2014. Hatchery spray cabinet administration does not damage avian coronavirus infectious bronchitis virus vaccine based on analysis by electron microscopy and virus titration. Avian Dis 59, 149-152, 144.

Sesti L, Sanguinetti HR, Zenobi CR, Jauregui MV, Chacon J, et al. 2014. A vaccine combination trial for the control of the variant $\mathrm{Q} 1$ infectious bronchitis virus (IBV) strain in South America. $8^{\text {th }}$ Symposium on Avian Corona and Pneumoviruses and Complicating Pathogens. Heffels-Redmann U, Lierz M, Enderlein D (eds). Rauischholzhausen, Germany, Pp 311-316.

Shepherd TA, Zhao Y, Li H, Stinn JP, Hayes MD, et al. 2015. Environmental assessment of three egg production systems - Part II. Ammonia, greenhouse gas, and particulate matter emissions. Poultry Sci 94, 534-543.

TAFS-Forum. 2011. World livestock disease atlas a quantitative analysis of global animal health data (2006-2009). The World Bank, Washington, DC, USA.

Tatar-Kis T, Kovacs EW, Felfoldi B, Nagy Z, Kiss I, et al. 2014. Protection achieved by a vaccination programme comprising Mass and 793B type vaccine against recent variant IBV strains circulating in the Middle East. 8th Symposium on Avian Corona and Pneumoviruses and Complicating Pathogens. Heffels-Redmann U, Lierz M, Enderlein D (eds). Rauischholzhausen, Germany, Pp 194-203. 
Toro H, van Santen VL, Li L, Lockaby SB, van Santen E, et al. 2006. Epidemiological and experimental evidence for immunodeficiency affecting avian infectious bronchitis. Avian Pathol 35, 455-464.

Toro H, Pennington D, Gallardo RA, van Santen VL, van Ginkel F, et al. 2012. Infectious bronchitis virus subpopulations in vaccinated chickens after challenge. Avian Dis 56, 501-508.

Toro H, van Santen VL, Jackwood MW. 2012. Genetic diversity and selection regulates evolution of infectious bronchitis virus. Avian Dis 56, 449-455.

Toro H. 2015. Monitoring chicken infectious anemia in commercial poultry. IDEXX Immunosupression Seminar, International Poultry Expo 201, Atlanta, GA, USA.

Valastro V, Holmes EC, Britton P, Fusaro A, Jackwood MW, et al. 2016 S1 gene-based phylogeny of infectious bronchitis virus: An attempt to harmonize virus classification. Inf Gen Evol 39, 349-364.

van Santen VL, Toro H. 2008. Rapid selection in chickens of subpopulations within ArkDPI-derived infectious bronchitis virus vaccines. Avian Pathol 37, 293-306.
Villegas P, Alvarado I. 2008. Virus identification and classification. A laboratory manual for the isolation, identification, and characterization of avian pathogens. Dufour-Zavala L, Glisson J, Jackwood MW, Pearson JE, Reed WM, Woolcock P (eds). American Association of Avian Pathologists, Pp 209-216.

Winterfield RW, Albassam MA. 1984. Nephropathogenicity of Infectious Bronchitis Virus. Poultry Sci 63, 2358-2363.

Zhao Y, Shepherd TA, Li H, Xin H. 2015. Environmental assessment of three egg production systems - Part I: Monitoring system and indoor air quality. Poultry Sci 94, 518-533.

Zhao Y, Zhao D, Ma H, Liu K, Atilgan A, et al. 2016. Environmental assessment of three egg production systems - Part III: Airborne bacteria concentrations and emissions. Poultry Sci 95, 1473-1481.

Ziebuhr J, Snijder EJ, Gorbalenya AE. 2000. Virus-encoded proteinases and proteolytic processing in the Nidovirales. J Gen Virol 81, 853-879. 